\title{
Derepression of the glyoxylate cycle in mutants of Neurospora crassa accelerated for growth on acetate
}

\author{
P. T. Chaure' ${ }^{1}$ and I. F. Connerton ${ }^{2}$ \\ Author for correspondence: I. F. Connerton. Tel: +44 1734 357000. Fax: +441734267917. \\ e-mail: IAN.CONNERTON@BBSRC.AC.UK
}

1 Department of

Microbiology, University of

Reading, Whiteknights,

PO Box 228,

Reading RG6 2AJ, UK

2 Institute of Food Research, Protein Engineering Department, Earley Gate, Whiteknights Road, Reading RG6 2EF, UK

\begin{abstract}
Two spontaneous allelic mutations have been isolated with the unusual semidominant phenotype of faster-than-wild-type growth on acetate as sole carbon source. The mutants were designated Aag-1 (accelerated acetate growth) and mapped on linkage group II. Upon re-isolation of both the Aag-1 alleles from repeated back-crosses to wild-type, between 1 and $6 \%$ of the progeny were found to be acu (acetate non-utilizing) mutants. Ten of these were selected for heterokaryon complementation analysis with known acu mutants; nine proved to be new alleles of acu-5 (deficient in acetyl-CoA synthetase), and one was a new acetate non-utilizing class, designated acu-14. Although the Aag-1 mutants clearly have no acetate-growth-related enzyme deficiencies, they did exhibit significant constitutive enzyme levels for acetylCoA synthetase and the glyoxylate cycle enzymes (isocitrate lyase and malate synthase) on the non-inducing carbon source, sucrose. The derepression was restricted to these enzymes, as representative enzymes from other carbonassimilatory pathways remained repressed and subject to carbon catabolite repression. Northern blot analysis of the mRNA levels of acetyl-CoA synthetase and the glyoxylate cycle enzymes from the mutants demonstrated the derepression to occur at the level of transcription. These data suggest that the physiological explanation for the accelerated acetate growth phenotype lies in the standing levels of the acetate-assimilatory enzymes, which enable the mutants to forgo some of the normal time required for adaption to growth on acetate.
\end{abstract}

Keywords: Neurospora crassa, ascomycete, glyoxylate cycle, acetate utilization

\section{INTRODUCTION}

Fungi utilize acetate as a sole carbon source via the action of acetyl-CoA synthetase. The resulting acetyl-CoA is incorporated into the glyoxylate cycle, which serves to replenish the tricarboxylic acid (TCA) cycle in the absence of glycolysis (Kornberg, 1966). This involves two reactions catalysed by the isocitrate lyase and malate synthase, which bypass the two decarboxylation steps of the TCA cycle. In addition to the enzymes of the glyoxylate cycle, phosphoenolpyruvate carboxykinase and $\mathrm{NADP}^{+}$-malic enzyme provide the metabolic links necessary for gluconeogenesis.

Abbreviations: RIP, repeat induced point mutation; TCA, tricarboxylic acid.
In a few species acetate non-utilization has been used as a criterion for the selection of biochemical mutants, including the filamentous ascomycetes Aspergillus nidulans (Armitt et al., 1976) and Neurospora crassa (Flavell \& Fincham, 1968a, b), together with the basidiomycete Coprinus cinereus (Casselton \& Casselton, 1974) and the yeast Yarrowia lipolytica (Barth, 1985). Flavell \& Fincham $(1968 \mathrm{a}, \mathrm{b})$ first isolated acetate non-utilizing mutants from $N$. crassa. These fell into seven distinct complementation groups mapping to seven independent loci. Three of these groups had single enzyme deficiencies: $a c u-3$ and $a c u-5$ lacked isocitrate lysase and acetyl-CoA synthetase, respectively, whereas acu-6 was deficient in phosphoenolpyruvate carboxykinase, which links gluconeogenesis with the primary acetate catabolic enzymes (Beever, 1975). Later, Rao \& Debusk (1977) reported an acetate nonutilizing permease-deficient mutant which was designated $a c p^{-i}$. 
Using mRNAs expressed during the adaptation of Neurospora mycelia from sucrose to acetate as hybridization probes, Thomas et al. (1988) isolated genomic clones involved in acetate utilization. Two of these clones were identified by their ability to complement the $N$. crassa acetyl-CoA synthetase mutant (acu-5) and the $A$. nidulans malate synthase mutant $(a c u E)$. A third clone was used to disrupt the resident gene using the RIP (Repeat Induced Point mutation) phenomenon of $N$. crassa and to generate a novel acu mutant, acu- 8 (Marathe et al., 1990). RIP is a pre-meiotic phenomenon in $N$. crassa, the effect of which is to cause extensive cytosine methylation and GC-AT transitions in duplicated DNA sequences (Selker et al., 1987). Therefore, transformed DNA sequences that duplicate resident genomic regions will trigger the process during a sexual cross and result in the disruption of the participating sequences. Later, $a c u-8$ was found to represent a null allele deficient in acetyl-CoA hydrolase (Connerton et al., 1992). Subsequently, the malatesynthase-deficient class was represented by RIP mutants and designated acu-9 (Connerton, 1990).

$N$. crassa mutants falling into a further four acetate nonutilizing complementation groups ( $a c u-10$ to $a c u-13$ ) were isolated by Owen et al. (1992) on the basis of resistance to the antimetabolite fluoroacetate. In the fellow ascomycete A. nidulans, three fluoroacetate-resistant/acetate nonutilizing classes have been isolated (Apirion, 1965). Of these, $\operatorname{fac} A$ is the structural gene for acetyl-CoA synthetase (Armitt et al., 1976; Connerton et al., 1990), and $f a c B$ has been shown to prevent normal induction of the glyoxylate cycle (Armitt et al., 1976; Katz \& Hynes, 1989). However, unlike the situation in $A$. nidulans none of the $N$. crassa fluoroacetate-resistant classes proved to be deficient in acetyl-CoA synthetase, or to have phenotypes affected in the regulation of the glyoxylate cycle. Analogous mutants to the latter regulatory class have also been reported in two other fungal species: the acu- 1 mutations of C. cinereus (Macanochie et al., 1992), and the GPR1 mutations of $Y$. lipolytica (Kujau et al., 1992). Two further mutants have been reported in $A$. nidulans that specifically cause the derepression of isocitrate lyase on non-inducing carbon sources (McCullough \& Roberts, 1980; McCullough \& Shanks, 1993). The $i c l^{c} A$ and $i c l^{c} B$ mutations act synergistically and are not affected by the presence of the fac $B$ mutation, implying that isocitrate lyase in $A$. nidulans is also subject to an alternative regulatory circuit independent of acetate induction.

It is perhaps surprising that none of the orthodox acetate non-utilizing Neurospora mutants have been shown to have regulatory effects on the expression of the glyoxylate cycle enzymes. In this paper we report the genetic and biochemical characterization of mutants of $N$. crassa that demonstrate a novel semi-dominant accelerated acetate growth phenotype and specific constitutive derepression of the glyoxylate cycle enzymes on non-inducing carbon sources such as sucrose.

\section{METHODS}

Strains. The $N$. crassa strains used in biochemical and heterokaryon tests were wild-type $74 A / a(\mathrm{ST} A / a)$ and $a c p^{-i}$ (Rao \&
Debusk, 1977); acu-1 (JI48), acu-3 (JI38), acu-5 (JI18; 94-78), acu-6 (JI31) and acu-7 (JI36) (Flavell \& Fincham, 1968a, b); acu8 (T27-1-8) (Marathe et al., 1990); acu-9 (T11-1) (Connerton, 1990); acu-10 (S10-3), acu-11 (S30-12), acu-12 (S10-4) and acu-13 (S30-9) (Owen et al., 1992); trp-3 (FGSC4093), pyr-4 (FGSC4030) and $A / a a^{132}$ (Kinnaird et al., 1982). The linkage group tester strain alcoy csp was used in initial mapping studies (Perkins, 1964): $A / a T(I ; I I) 4637$ al-1; T(IV; V)R2355, cot-1; $T(I I I ; V I) 1, y l o-1 ;$ csp-2 (FGSC3661/3434). The secondary testers for linkage-group determination were LGI and LGII $a$ al-1, arg-5 (FGSC1206); LGIV and LGV a cot-1, al-3 (FGSC1244); LGIII and LGVI $a$ trp-1, ylo-1 (FGSC1208); and LGVII a nic-3, met-7, arg-10 (FGSC4142).

Media. Vogel's minimal medium (Vogel, 1956) was supplemented with $2 \%(\mathrm{w} / \mathrm{v})$ sucrose or with one of the following alternative carbon sources: $40 \mathrm{mM}$ sodium acetate, $40 \mathrm{mM}$ ethanol, $40 \mathrm{mM}$ quinate, $0.5 \%$ Avicel, $0.5 \%$ oatspelt xylan. Solid medium contained $1.5 \%(\mathrm{w} / \mathrm{v})$ agar (Difco), $0.5 \%(\mathrm{w} / \mathrm{v})$ fructose and glucose $(\mathrm{BDH})$ as carbon source together with $2 \%$ (w/v) L-sorbose (Sigma) for induction of colonial growth (Tatum et al., 1949). When using acetate as carbon source colonial growth was induced with $0 \cdot 2 \%$ L-sorbose. Vogel's fluoroacetate medium contained $0.5 \mathrm{M}$ sodium fluoroacetate (Aldrich/Sigma) supplemented with $0.5 \%$ fructose and glucose and $0.2 \%$ sorbose. Tryptophan $(1 \mathrm{mM})$, uracil/uridine $(3 \mathrm{mM})$ and glutamate $(3 \mathrm{mM})$ were used as supplements for the $\operatorname{tr} p-3$, pyr-4 and am mutants, respectively. Vogel's medium supplemented with $20 \mathrm{mM}$ glycine was used to counter-select am mutants. Methionine ( $1 \mathrm{mM})$, arginine $(1 \mathrm{mM})$ and niacinamide $\left(2 \mu \mathrm{g} \mathrm{ml}^{-1}\right)$ were used as supplements for the linkage group VII markers.

Growth tests. A loopful of conidia was plated onto agar containing the appropriate growth medium including the paramorphogen sorbose to produce colonial growth. Growth was normally scored after $3 \mathrm{~d}$ incubation at $30^{\circ} \mathrm{C}$. However, when scoring the accelerated acetate growth phenotype the plates were checked at 1,2 and $3 \mathrm{~d}$ to ensure the demarcation between mutant and wild-type strains. Growth was scored following overnight incubation at $30^{\circ} \mathrm{C}$ when using media without sorbose, except when scoring for cot, when $37^{\circ} \mathrm{C}$ was used. For scoring in liquid medium, $0.1 \mathrm{ml}$ of a $10^{6} \mathrm{ml}^{-1}$ conidial suspension was used to inoculate $5 \mathrm{ml}$ medium, and growth was scored after standing for 2-3 d. Growth curves were generated by inoculating multiple $50 \mathrm{ml}$ aliquots of Vogel's sucrose or acetate medium with approximately $10^{5}$ conidia and harvesting duplicate samples at timed events. The mycelial yields of these samples were determined as dry weights and averaged.

Crossing and revival of ascospores. $1 \times$ Westergaard and Mitchell's crossing medium (Westergaard \& Mitchell, 1947) was used for sexual crosses. Petri dishes containing discs of 3MM Whatman filter paper (cellulose) as a carbon source were used to increase the fertility of crosses. Ascospores were collected from the Petri dish lid after release and after heatshock at $60^{\circ} \mathrm{C}$ they were germinated on Vogel's sucrose medium plus the appropriate supplements. A random sample of germinated ascospores was selected and transferred to slopes of a similar medium and incubated further until abundant conidia were present. Conidia from each isolate were then tested for their ability to grow on selective media with sorbose.

Complementation tests. Drops of dense conidial suspensions of the mutants to be tested were added to $5 \mathrm{ml}$ liquid medium and incubated at $30^{\circ} \mathrm{C}$ until many new conidia had formed on the mycelial growth. Of these new conidia some were presumed to be heterokaryotic and were then tested for their ability to 
grow on appropriate media. Single colonies of these could be picked and their growth on further selective media was scored.

Enzyme assays. Enzyme activities were determined from crude extracts of wild-type and mutant strains. Mycelial pads were grown in $1 \times$ Vogel's sucrose medium for $16 \mathrm{~h}$ and induced for $8 \mathrm{~h}$ on inducing medium at $30^{\circ} \mathrm{C}$ with shaking. Crude enzyme extracts were then prepared by grinding the mycelial pads with chilled $0.05 \mathrm{M}$ sodium phosphate buffer ( $\mathrm{pH} \mathrm{7.2)}$ and acidwashed sand. Supernatants were retrieved after centrifugation in a microcentrifuge for $1 \mathrm{~min}$ at 13000 r.p.m. and stored on ice for use in enzyme assays and protein determination. Protein concentrations were determined using Coomassie Brilliant Blue G-25 dye-binding. Reagent volumes were used such as to enable the assay to be run in a microtitre plate in $200 \mu \mathrm{l}$ aliquots using an ELISA plate reader calibrated at $720 \mathrm{~nm}$. The subsequent enzyme assays were done as described by Dixon \& Kornberg (1959) and detailed in Owen et al. (1992). Glucosidase and xylanase assays were performed on culture supernatants following $4 \mathrm{~d}$ growth on Avicel or oat-spelt xylan.

Northern transfers. RNA was extracted from $N$. crassa mycelium according to the method of Sokolovsky et al. (1990) using $\mathrm{LiCl}$. RNA was electrophoresed in formaldehyde denaturing agarose gels according to the method in Maniatis $e t$ al. (1982) with $1 \times$ MOPS, blotted on to Hybond-N nylon filters (Amersham) and hybridized to labelled ('Multiprime labelling'; Amersham) DNA probes. The resulting autoradiographs obtained with different radiolabelled probes were scanned using a laser densitometer and scaled to each other. The intensities of the relative signals were then recorded.

\section{RESULTS}

\section{Isolation of mutants}

To check the culture medium and the wild-type parent (ST $A$ ) for a routine cross, a conidial suspension was plated out in serial dilutions onto Vogel's acetate containing $0 \cdot 2 \%$ L-sorbose. This medium usually supports visible colonial growth of acetate-utilizing strains after $3 \mathrm{~d}$ at $30^{\circ} \mathrm{C}$. Unexpectedly, a vigorously growing colony appeared after $24 \mathrm{~h}$, and other colonies appeared after 2-3 d growth. The colony was picked and subcultured on Vogel's sucrose medium and re-tested for growth on acetate plates, where it continued to grow faster than several acetate-utilizing laboratory strains. Approximately $10^{5}$ conidia of wild-type stock were then plated as dense suspensions on Vogel's acetate and observed for vigorous growth after $24 \mathrm{~h}$ in an attempt to isolate further mutants with similar phenotypes. This procedure yielded only one further potential mutant after screening $10^{7}$ conidia. Comparative growth curves for these strains (Fig. 1) revealed that both grew faster than wild-type on acetate medium. In addition, the putative mutants appeared disadvantaged compared with the wild-type during growth on sucrose.

\section{Genetic characterization}

The primary mutations were initially re-isolated from a back-cross to wild-type, almost half of the progeny from which exhibited the accelerated acetate growth (Aag) phenotype as would be expected for a single mutation.

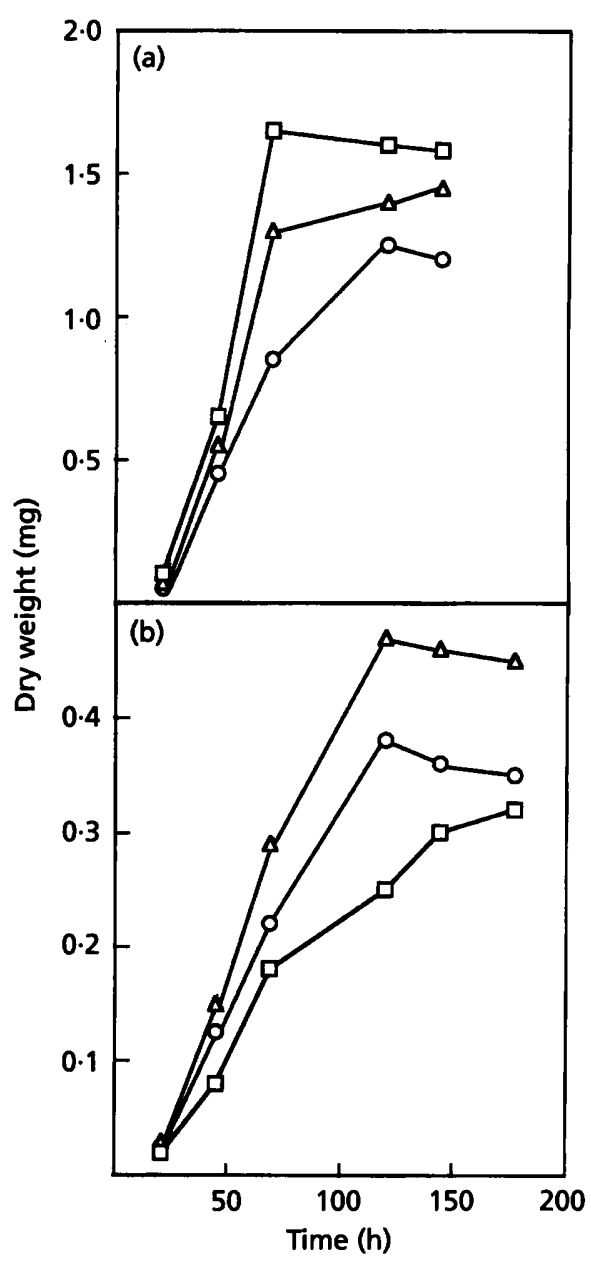

Fig. 1. Growth of wild-type ( $\square)$, Aag-1-1 (T13) ( $\triangle$ ) and Aag-1-2 (T14) (O) from similar conidial inocula on equivalent amounts of sucrose (a) or acetate (b) in shaken cultures.

However, a number of these progeny failed to grow at all on acetate plates. In a further out-cross to $a m^{\mathbf{1 3 2}}$ to generate double mutants for heterokaryon complementation tests, both fast-acetate-growth mutations gave rise to a few acetate non-utilizing progeny. Five of these were selected from each cross and tested for heterokaryotic complementation on liquid acetate medium with all the acetate non-utilizing classes available in the laboratory (acu-1, -3, -5, -6, -7, -8, -9, -10, -11, -12, -13 and $\left.a c p^{i}\right)$. All but one of the acu strains proved to be new alleles of $a c u-5$, the structural gene for the primary acetateactivating enzyme acetyl-CoA synthetase. The single exception to this, designated acu-14, was able to complement all of the known mutations and was therefore considered to represent a new acetate non-utilizing class.

The am Aag double mutants re-isolated from the outcrosses were combined in heterokaryons with $\operatorname{trp}-3$ and pyr-4 strains. Heterokaryons from both Aag strains with both auxotrophic partners demonstrated faster-than-wildtype growth on acetate plates, although perhaps not to the same degree as the initial mutants. It would appear that 
both mutations have semi-dominant characteristics. Representative strains of both mutants of opposite mating type were then crossed and their progeny were tested for growth on acetate. Again a few of these turned out to be acetate non-utilizers; however, the rest retained the $\mathrm{Aag}^{+}$ phenotype. As there were no wild-type recombinants from this cross, the mutations were considered to be allelic representatives of a single gene (Aag1-1 and Aag12). To demonstrate further that the mutations represent a common gene and to map the new locus, Aag1-1 and Aag1-2 were crossed to the triple translocation strain alcoy csp (Perkins, 1964). The alcoy csp strains bear the visible markers albino (white conidia), cot-1 (temperaturesensitive colonial growth) and ylo (yellow conidia which are epistatic to white), which identify the six linkage groups involved in the interchanges, and $c s p-2$ (conidial separation), which identifies the remaining independent linkage group (LGVII). Over 100 ascospore progeny that were recovered from these crosses were scored for the visible markers and the fast acetate growth phenotype. Both crosses indicated loose linkage to csp-2 on LGVII, although spore germination was notably low in these crosses $(<50 \%)$. Recombination frequencies with all the other markers did not differ significantly from $50 \%$. To confirm the preliminary assignment of the Aag-1 mutation to LGVII, the Aag-1-1 allele was crossed with a secondary linkage group tester, FGSC 4142, which carries the nic-3, met-7 and arg-10 markers. However, Aag-1 did not demonstrate significant linkage to the nic-3 $(44 \cdot 6 \%)$, met$7(46.5 \%)$ and $\arg -10(48 \%)$ markers. Aag- 1 was therefore crossed independently with three further tester strains carrying markers representing the remaining linkage groups (al-1 arg-5, FGSC 1206; cot-1 al-3, FGSC 1244; trp1 ylo-1, FGSC 1208). In these crosses, linkage was only observed with the arg-5 marker (33.0\%) located on LGII.

As the Aag-1 mutations were repeatedly found to produce new alleles of $a c u-5$ in spore progeny and were found to map to the same linkage group as the acu-5 gene, it is possible that the Aag-1 isolates were either initially double mutants harbouring a closely linked functionally unstable allele of $a c u-5$ or were unstable alleles of $a c u-5$ itself conferring a pleiotropic phenotype. To investigate these possibilities, mutant Aag-1-1 was crossed to an independent allele of $a c u-5$ (JI18) and the spore progeny were tested for growth on acetate medium. The cross yielded $12.9 \%(18 / 140)$ wild-type progeny, implying a recombination frequency of $26 \%$ as the Aag- 1 mutants will be epistatic to $a c u-5$ (JI18) in the reciprocal class. The acu class $(68 / 140)$ were bolstered by a similar number (19) to the wild-type class over the fast-acetate-growth class (49/140). These data confirm that the Aag- 1 and the acu5 genes are on the same linkage group but are not allelic nor are they particularly closely linked.

\section{Biochemical properties}

Initially mycelial extracts of the Aag-1 mutations prepared following growth on sucrose (non-induced) and subsequent growth on acetate (induced) were assayed for the enzymes of the glyoxylate cycle, gluconeogenesis and
Table 1. Enzyme activities of wild-type $N$. crassa and the Aag-1 mutants on various carbon sources

Specific activities were measured in cell-free extracts after $16 \mathrm{~h}$ growth on sucrose medium (non-induced) and following a subsequent $8 \mathrm{~h}$ incubation in acetate, ethanol, quinate, cellulose or xylan (induced). Values represent the means of at least two sets of duplicate assays. NT, Not tested.

\begin{tabular}{|c|c|c|c|c|c|c|}
\hline \multirow[t]{2}{*}{ Strain } & \multicolumn{6}{|c|}{ Enzyme activity $\left(\mu \mathrm{mol} \mathrm{min}^{-1} \mathrm{mg}^{-1}\right.$ ) on: } \\
\hline & Sucrose & Acetate & Ethanol & Quinate & Cellulose & Xylan \\
\hline \multicolumn{7}{|c|}{ Acetyl-CoA synthetase } \\
\hline $\mathrm{ST} A$ & $0 \cdot 132$ & $0 \cdot 463$ & $0 \cdot 283$ & $0 \cdot 282$ & $0 \cdot 122$ & NT \\
\hline T13 & 0.526 & $0 \cdot 914$ & $0 \cdot 316$ & $0 \cdot 437$ & $0 \cdot 114$ & NT \\
\hline T14 & 0.476 & 0.997 & $0 \cdot 383$ & $0 \cdot 402$ & $0 \cdot 146$ & NT \\
\hline \multicolumn{7}{|c|}{ Isocitrate lyase } \\
\hline ST $A$ & 0.35 & $2 \cdot 81$ & $0 \cdot 92$ & $0 \cdot 76$ & $0 \cdot 22$ & $0 \cdot 21$ \\
\hline T13 & $0 \cdot 91$ & $12 \cdot 83$ & $3 \cdot 83$ & $1 \cdot 83$ & $0 \cdot 85$ & $0 \cdot 81$ \\
\hline T14 & $2 \cdot 22$ & $8 \cdot 75$ & $1 \cdot 83$ & $2 \cdot 56$ & $0 \cdot 14$ & $1 \cdot 62$ \\
\hline \multicolumn{7}{|c|}{ Malate synthase } \\
\hline $\mathrm{ST} A$ & $0 \cdot 31$ & $1 \cdot 82$ & $1 \cdot 02$ & $1 \cdot 42$ & NT & NT \\
\hline T13 & $0 \cdot 88$ & $3 \cdot 13$ & $2 \cdot 33$ & $2 \cdot 11$ & NT & NT \\
\hline T14 & $0 \cdot 95$ & $2 \cdot 37$ & $2 \cdot 19$ & 1.98 & NT & NT \\
\hline \multicolumn{7}{|c|}{ Phosphoenolpyruvate carboxykinase } \\
\hline $\mathrm{ST} A$ & $0 \cdot 009$ & $0 \cdot 039$ & $0 \cdot 019$ & $0 \cdot 021$ & $0 \cdot 001$ & $0 \cdot 001$ \\
\hline T13 & $0 \cdot 006$ & $0 \cdot 032$ & $0 \cdot 021$ & $0 \cdot 023$ & 0.006 & $0 \cdot 004$ \\
\hline T14 & $0 \cdot 011$ & $0 \cdot 029$ & $0 \cdot 018$ & $0 \cdot 022$ & 0.001 & 0.001 \\
\hline \multicolumn{7}{|c|}{ Fructose-1,6-biphosphatase } \\
\hline $\mathrm{ST} A$ & $0 \cdot 28$ & $0 \cdot 35$ & $0 \cdot 33$ & $0 \cdot 38$ & $0 \cdot 11$ & $0 \cdot 16$ \\
\hline T13 & $0 \cdot 25$ & $0 \cdot 39$ & $0 \cdot 29$ & $0 \cdot 35$ & $0 \cdot 13$ & $0 \cdot 18$ \\
\hline T14 & $0 \cdot 28$ & 0.35 & $0 \cdot 31$ & $0 \cdot 36$ & $0 \cdot 21$ & 0.21 \\
\hline \multicolumn{7}{|c|}{ Alcohol dehydrogenase } \\
\hline $\mathrm{ST} A$ & $0 \cdot 054$ & 0.068 & $0 \cdot 225$ & $0 \cdot 057$ & $0 \cdot 074$ & 0.052 \\
\hline T13 & 0.053 & 0.052 & $0 \cdot 305$ & $0 \cdot 062$ & $0 \cdot 111$ & $0 \cdot 139$ \\
\hline $\mathrm{T} 14$ & 0.063 & 0.071 & $0 \cdot 266$ & 0.061 & $0 \cdot 055$ & 0.058 \\
\hline
\end{tabular}

acetate mobilization (Table 1). It is apparent that the primary acetate-utilizing enzymes (acetyl-CoA synthetase, isocitrate lyase and malate synthase) were all elevated compared to the wild-type under non-inducing conditions. To examine whether the observed derepression was unique to the acetate assimilatory enzymes or was characteristic of other carbon catabolic pathways, a number of catabolic enzyme levels were measured under repressed and derepressed conditions. It is clear from these measurements (Table 1) that none of the other pathways examined are subject to derepression in the Aag-1 mutants. As the repressed levels of acetyl-CoA synthetase were abnormally high, it seemed likely that the Aag-1 mutants would show increased sensitivity to the antimetabolite fluoroacetate. Fluoroacetate undergoes lethal synthesis via fluoroacetyl-CoA to fluorocitrate and poisons aconitase. The Aag-1 mutants were indeed only tolerant up to $0 \cdot 20 \mathrm{M}$ whereas the wild-type could tolerate $0 \cdot 35 \mathrm{M}$ fluoroacetate.

To ascertain whether the observed derepressed levels of 
(a)

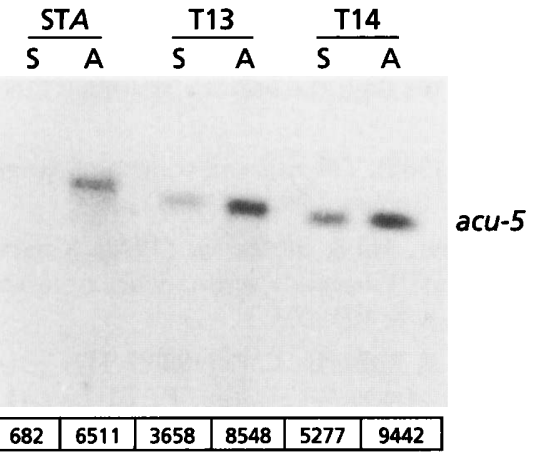

(b)

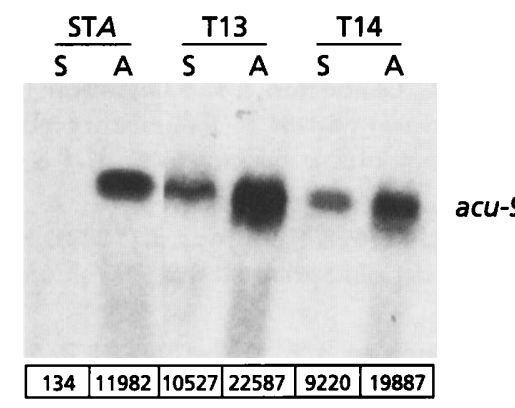

(c)

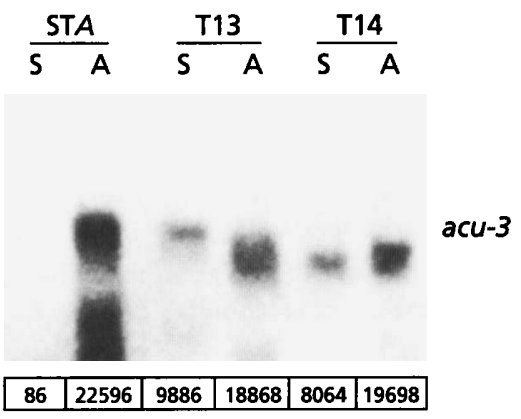

(d)

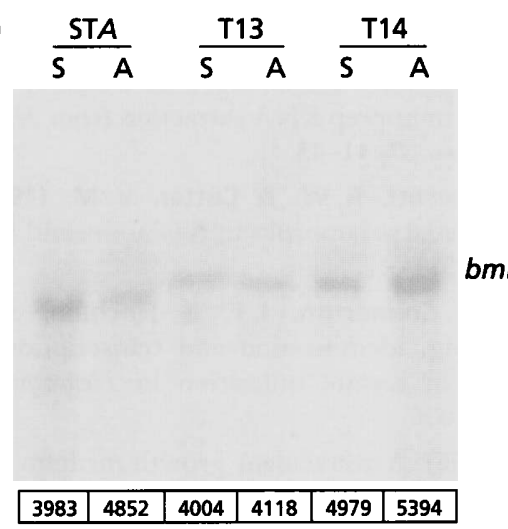

Fig. 2. Northern blot analysis of wild-type and the Aag-1-1 (T13) and Aag-1-2 (T14) alleles. Total RNAs were isolated after $16 \mathrm{~h}$ growth on sucrose (S) or following $6 \mathrm{~h}$ induction on acetate $(A)$. Independent Northern transfers were probed with (a) $6 \mathrm{~kb}$ HindlII fragment of the acu-5 acetyl-CoA synthetase gene, (b) $1.7 \mathrm{~kb} \mathrm{BamHI}$ fragment of the acu-9 malate synthase gene, (c) $1.2 \mathrm{~kb}$ Hindlll fragment of the acu-3 gene and (d) $3.1 \mathrm{~kb}$ HindlII fragment of the $\mathrm{bml} \beta$-tubulin gene as a constitutive mRNA control. Aliquots (approx. $20 \mu \mathrm{g}$ ) of the RNA preparations were initially probed for $\beta$-tubulin and the RNA quantities were standardized in repeated blots using a laser densitometer according to the observed signal. The boxed figures at the bottom of each part represent the signal strength in pixels. the acetate-responsive enzymes were due to the release of otherwise inactive enzymes (translational control) or due to inopportune mRNA synthesis (transcriptional control) Northern transfers were undertaken. Fig. 2 shows the relative mRNA levels for the wild-type and Aag-1 mutant strains after mycelial growth on sucrose and acetate of the acetate responsive structural genes for acetyl-CoA synthetase (acu-5), isocitrate lyase (acu-3) and malate synthase (acu-9). It is clear that compared with the wild-type both of the Aag-1 alleles demonstrate marked transcriptional derepression on sucrose.

\section{DISCUSSION}

The initial fortuitous selection of a spontaneous mutant of $N$. crassa able to produce fast vigorous growth on acetate plates provided the basis for the selection of potentially novel mutants. Subsequent selection for further mutants of this class, leading to one further mutant which proved to be allelic, demonstrated the mutation to be rare. Indicative of their potential as regulatory mutants, both Aag-1 alleles gave rise to the semi-dominant phenotype of faster-than-wild-type growth on acetate as sole carbon source. However, both had the physiological disadvantage of slower-than-wild-type growth on the preferred carbon source, sucrose. The Aag-1 mutants demonstrated the specific derepression of the acetate inducible enzymes acetyl-CoA synthetase, isocitrate lyase and malate synthase on the normally repressing carbon source, sucrose. Enzymes in other carbon-assimilatory pathways were unaffacted, as were the gluconeogenic enzymes coinduced by acetate, phosphoenolpyruvate carboxykinase and fructose-1,6-biphosphatase. A simple physiological explanation of the growth phenotype appears to be that the standing levels of the acetate-assimilatory enzymes enable the mutants to utilize the new carbon source quicker than the wild-type. Following transfer to acetate the mutants also demonstrate enzyme induction to levels greater than in the wild-type. Acetyl-CoA synthetase levels are normally carefully regulated in the cell because excess cytosolic acetyl-CoA can be toxic. Acetyl-CoA synthetase levels are regulated not only under conditions of catabolite repression but also following induction, as the enzyme and corresponding mRNA levels do not approach those observed for the co-inducible glyoxylate cycle enzymes. The cytosolic acetyl-CoA concentration is further modulated by a second co-inducible enzyme, acetyl-CoA hydrolase (Connerton et al., 1992), to prevent possible toxic accumulation. The levels of acetyl-CoA synthetase in the Aag-1 mutants are therefore a potential source of physiological stress. To relieve this stress the Aag-1 mutation must either revert or force a second site mutation. When growth on acetate is not selected, a prime candidate for this is the structural gene for acetyl-CoA synthetase. The observation that a variable quotient of homokaryotic ascopore progeny are acu-5 alleles may reflect this.

Messenger RNA levels of the acetate-responsive genes, detected on Northern transfers, demonstrated the abnormal derepression of these genes during growth on sucrose. The derepression is clearly due to a loss of 
transcriptional control. Two types of mutational event may directly cause transcriptional derepression: (i) a mutation leading to the loss or dysfunction of a repressor protein, or (ii) a missense mutation in an activator protein such that it can no longer be recognized directly by its cognate repressor or it can no longer be displaced from its target promoter binding site. As the Aag-1 mutants are semi-dominant, (i) is not compatible and can therefore be discounted. A mutation in the putative activator would have a dominant phenotype.

\section{ACKNOWLEDGEMENTS}

P.T.C. was the recipient of an overseas scholarship from the Indian Government.

\section{REFERENCES}

Apirion, D. (1965). The two way selection of mutants and revertants in respect of acetate utilization and resistance to fluoroacetate in Aspergillus nidulans. Genet Res 6, 317-329.

Armitt, S., McCullough, W. \& Roberts, C. R. (1976). Analysis of acetate non-utilizing (acu) mutants in Aspergillus nidulans. $J$ Gen Microbiol 92, 263-282.

Barth, G. (1985). Genetic regulation of isocitrate lyase in the yeast Yarrowia lipolytica. Curr Genet 10, 119-124.

Beever, R. E. (1975). Regulation of 2-phosphoenolpyruvate kinase and isocitrate lyase in Neurospora crassa. J Gen Microbiol 86, 197-200.

Casselton, L. A. \& Casselton, P. J. (1974). Functional aspects of fluoroacetate resistance in Coprinus with special reference to acetylCoA synthetase deficiency. Mol \& Gen Genet 132, 255-264.

Connerton, I. F. (1990). Premeiotic disruption of the Neurospora crassa malate synthase gene by native and divergent DNAs. Mol \& Gen Genet 223, 319-323.

Connerton, I. F., Fincham, J. R. S., Sandeman, R. A. \& Hynes, M. J. (1990). Comparison and cross-species expression of the acetyl-CoA synthetase genes of the ascomycete fungi, Aspergillus nidulans and Neurospora crassa. Mol Microbiol 4, 451-460.

Connerton, I. F., McCullough, W. \& Fincham, J. R. S. (1992). An acetate-sensitive mutant of Neurospora crassa deficient in acetyl-CoA hydrolase. J Gen Microbiol 138, 1797-1800.

Dixon, G. H. \& Kornberg, H. L. (1959). Assay methods for key enzymes of the glyoxylate cycle. Biochem $J$ 72, 3P.

Flavell, R. B. \& Fincham, J. R. S. (1968a). Acetate non-utilizing mutants of Neurospora crassa. 1. Mutant isolation, complementation studies and linkage relationships. J Bacteriol 95, 1056-1062.

Flavell, R. B. \& Fincham, J. R. S. (1968b). Acetate non-utilizing mutants of Neurospora crassa. 2. Biochemical deficiencies and roles of certain enzymes. $J$ Bacteriol 95, 1063-1068.

Katz, M. E. \& Hynes, M. J. (1989). Isolation and analysis of the acetate regulatory gene $f a c B$, from Aspergillus nidulans. Mol Cell Biol 12, 5696-5701.
Kinnaird, J. H., Keighen, M. A., Kinsey, J. A. \& Fincham, J. R. S. (1982). Cloning of the am (glutamate dehydrogenase) gene of Neurospora crassa through the use of a synthetic DNA probe. Gene 20, 387-396.

Kornberg, H. L. (1966). The role and control of the glyoxylate cycle in Escherichia coli. Biochem J 99, 1-11.

Kujau, M., Weber, H. \& Barth, G. (1992). Characterization of mutants of the yeast Yarrowia lipolytica deficient in acetyl-coenzyme A synthetase. Yeast 8, 193-203.

McCullough, W. \& Roberts, C. F. (1980). The genetic control of isocitrate lyase in Aspergillus nidulans. FEBS Lett 41, 238-242.

McCullough, W. \& Shanks, A. (1993). Properties of genes involved in the control of isocitrate lyase production in Aspergillus nidulans. $J$ Gen Microbiol 139, 509-511.

Maconochie, M. K., Connerton, I. F. \& Casselton, L. A. (1992). The acu-1 gene of Coprinus cinereus is a regulatory gene required for induction of acetate utilisation enzymes. Mol \& Gen Genet 234, 211-216.

Maniatis, T., Fritsch, E. F. \& Sambrook, J. (1982). Molecular Cloning: a Laboratory Manual. Cold Spring Harbor, NY: Cold Spring Harbor Laboratory.

Marathe, S., Connerton, I. F. \& Fincham, J. R. S. (1990). Duplication-induced mutation of a new Neurospora gene required for acetate metabolism: properties of the mutant and predicted amino acid sequences of the protein product. Mol Cell Biol 10, 2638-2644.

Owen, N. E., Chaure, P. T. \& Connerton, I. F. (1992). Isolation and characterization of new fluoroacetate/acetate non-utilizing mutants of Neurospora crassa. J Gen Microbiol 138, 2599-2608.

Perkins, D. D. (1964). Multiple interchange stocks for linkage detection. Neurospora Newsl 6, 22-23.

Rao, T. K. \& Debusk, A. G. (1977). An inducible acetate transport system in Neurospora crassa conidia. Biocbim Biophys Acta 470, 475-483.

Selker, E. U., Cambereri, E. B., Jensen, B. C. \& Haack, K. R. (1987). Rearrangement of duplicated DNA in specialized cells of Neurospora. Cell 51, 741-752.

Sokolovsky, V., Kaldenhoff, R., Ricci, M. \& Russo, V. E. A. (1990). Fast and reliable mini-prep RNA extraction from Neurospora crassa. Fungal Genet Newsl 37, 41-43.

Tatum, E. L., Barratt, R. W. \& Cutter, V. M. (1949). Chemical induction of colonial paramorphs in Neurospora and Syncephelastrum. Science 109, 509-511.

Thomas, G. H., Connerton, I. F. \& Fincham, J. R. S. (1988). Molecular cloning, identification and transcriptional analysis of genes involved in acetate utilization in Neurospora crassa. Mol Microbiol 2, 599-606.

Vogel, H. J. (1956). A convenient growth medium for Neurospora (Medium N). Microb Genet Bull 13, 42-43.

Westergaard, M. \& Mitchell, H. K. (1947). A synthetic medium favouring sexual reproduction. Am J Bot 34, 573-577.

Recived 11 October 1994; accepted 20 February 1995. 